\title{
Aspergilosis pulmonar necrotizante crónica como complicación de silicosis
}

\section{Chronic necrotizing pulmonary aspergillosis as a complication of silicosis}

\author{
J.J. Blanco ${ }^{1}$, F.J. González Barcala², M.A. Álvarez Moure ${ }^{3}$, M.C. González Mao ${ }^{4}$, \\ E. Temes ${ }^{1}$, J.L. Guerra ${ }^{1}$
}

\section{RESUMEN}

La aspergilosis pulmonar necrotizante crónica es una rara enfermedad que tiende a afectar a personas con enfermedad pulmonar subyacente o inmunocomprometidos en grado leve. Es una complicación excepcional de la silicosis y su diagnóstico precisa un alto índice de sospecha pues simula otras enfermedades como tuberculosis y neoplasia, complicaciones más frecuentes en estos pacientes. Presentamos tres casos con larga historia de exposición a sílice y diagnóstico de fibrosis masiva progresiva que presentaron aspergilosis pulmonar. Revisamos sus características clínicas, radiológicas y tratamiento.

Palabras clave. Silicosis. Aspergilosis pulmonar necrotizante crónica. Diagnóstico. Tratamiento.

\begin{abstract}
Chronic necrotizing pulmonary aspergillosis is un uncommon disease which is found in people with underlying lung disease or in mildly immunocompromised patients. It is an exceptional complication in silicosis and its diagnosis is difficult because it simulates other diseases like tuberculosis and cancer which are more common in such patients. We report on three cases with a long history of silica dust exposure with pulmonary aspergillosis complicating progressive massive fibrosis (PMF). We review their clinical, radiological and treatment features.
\end{abstract}

Key words. Silicosis. Chronic necrotizing pulmonary aspergillosis. Diagnostic. Treatment.
1. Servicio de Neumoloxía. Hospital Meixoeiro. Complexo Hospitalario Universitario de Vigo.

2. Servicio de Neumología. Hospital de Montecelo. Complexo Hospitalario de Pontevedra.

3. Servicio de Radiodiagnóstico. Hospital Meixoeiro. Complexo Hospitalario Universitario de Vigo.

4. Servicio de Análisis clínicos. Hospital Meixoeiro. Complexo Hospitalario Universitario de Vigo.

\section{Correspondencia}

José Jesús Blanco Pérez

S. Miguel de Tabagón. O Rosal

36760 Pontevedra

E-mail: josejesus.blanco.perez@sergas.es

Recepción: 2 de febrero de 2011

Aceptación provisional: 14 de febrero de 2011

Aceptación definitiva: 17 de febrero de 2011 


\section{INTRODUCCIÓN}

El Aspergillus es un hongo formador de esporas, ubicuo, ampliamente distribuido sobre todo en medio húmedo. Existen numerosas especies, pero solo 20 causan enfermedad siendo los más comunes: $A$. fumigatus (90\%), A. flavus, $A$. terreus, $A$. niger y A. nidulans ${ }^{1}$. Las esporas, con un tamaño de 2 a $3 \mu \mathrm{m}$ de diámetro pueden impactarse en las vías aéreas terminales. Por su capacidad de adherirse a superficies epiteliales y endoteliales y por su tendencia a invadir vasos sanguíneos, puede causar invasión pulmonar ${ }^{2}$. Dada su baja patogenecidad se pueden inhalar cientos de esas esporas diariamente, pero solo unas pocas personas desarrollan la enfermedad ${ }^{2}$. Una vez inhalado, el hongo puede causar una variedad de síndromes $\operatorname{clínicos}^{3}$, de acuerdo a la arquitectura del pulmón, la respuesta inmune del huésped y la propia virulencia del hongo, así aspergilosis broncopulmonar alérgica (ABPA), aspergilosis invasiva y las formas de aspergilosis pulmonar crónica: aspergiloma simple, pelota de hongos que coloniza una cavidad preexistente, aspergilosis pulmo- nar crónica cavitaria (APCC) y aspergilosis semiinvasiva o aspergilosis pulmonar necrotizante crónica APNC. El término de APNC se reserva para pacientes con algún grado de inmunosupresión, con una duración de la enfermedad más corta a diferencia de la APCC 4 . A principios de los años ochenta Binder y col $^{5}$ propusieron el término de APNC y Gefter y col ${ }^{6}$ el de aspergilosis semiinvasiva para una forma destructiva indolente secundaria a invasión local del parénquima pulmonar por especies de Aspergillus. Un examen patológico demostrando invasión tisular confirma la enfermedad, pero esto es frecuentemente difícil de obtener, por lo cual el diagnóstico acostumbra a hacerse ante un contexto clínico-radiológico compatible y cultivo positivo para Aspergillus ${ }^{5,7}$. Aunque escasamente descrito, el antecedente de neumoconiosis se considera factor de riesgo para APNC ${ }^{5,8}$. Desde 1994 hasta 2010 han sido hospitalizados 356 pacientes con el diagnóstico de silicosis en nuestro hospital y 3 de ellos cuyas características clínicas y microbiológicas se muestran (Tabla 1) han presentado esta rara complicación.

Tabla 1. Características clínicas y microbiológicas de los 3 casos (FMP: fibrosis masiva progresiva)

\begin{tabular}{|c|c|c|c|}
\hline & Paciente 1 & Paciente 2 & Paciente 3 \\
\hline Edad & 45 & 44 & 45 \\
\hline E. Previa & $\begin{array}{l}\text { FMP } \\
\text { tabaquismo } \\
\text { prednisona } \geq 10 \mathrm{mg} / \text { día }\end{array}$ & $\begin{array}{l}\text { FMP } \\
\text { etilismo } \\
\text { tabaquismo }\end{array}$ & $\begin{array}{l}\text { FMP } \\
\text { tabaquismo }\end{array}$ \\
\hline TBP & Sí & Sí & Sí \\
\hline Síntomas & $\begin{array}{l}5 \text { meses } \\
\text { disnea y ex. hemoptoica }\end{array}$ & $\begin{array}{l}2 \text { meses } \\
\text { hemoptisis y síndrome } \\
\text { general }\end{array}$ & $\begin{array}{l}1 \text { mes } \\
\text { tos y hemoptisis }\end{array}$ \\
\hline TAC & $\begin{array}{l}\text { Conglomerados } \\
\text { Cavitación } \\
\text { Micetoma }\end{array}$ & $\begin{array}{l}\text { Cavitación } \\
\text { Micetoma }\end{array}$ & $\begin{array}{l}\text { Conglomerados } \\
\text { Cavitación } \\
\text { Micetoma }\end{array}$ \\
\hline Precipitinas & + & + & + \\
\hline Galactomanano & - & + & - \\
\hline Especie Aspergillus & A. fumigatus & A. flavus & A. fumigatus \\
\hline Tratamiento & Voriconazol & $\begin{array}{l}\text { Itraconazol 3meses } \\
\text { Voriconazol }\end{array}$ & Voriconazol \\
\hline
\end{tabular}




\section{OBSERVACIÓN CLÍNICA}

\section{Caso 1}

Varón de 45 años, trabajador en canteras durante 23 años, diagnosticado de silicosis complicada (FMP). Exfumador desde hacía 10 años de 15 paquetes/año y tuberculosis pulmonar (TBP) en el año 2000 tratada correctamente con recidiva en 2003. Había precisado cursos frecuentes de esteroides en los 2 últimos años. Acudió al Servicio de Urgencias por disnea y expectoración hemoptoica de 5 meses de evolución. En la exploración física presentaba febrícula $\left(37,3^{\circ} \mathrm{C}\right)$. La auscultación pulmonar mostraba roncus y sibilantes bilaterales. En la analítica destacaban una hemoglobina de 11,9 g/l; plaquetas: 515.000 / $\mathrm{mm}^{3}$; velocidad de sedimentación $79 \mathrm{~mm} / \mathrm{h}$ y proteína $\mathrm{C}$ reactiva de $50 \mathrm{mg} / \mathrm{l}$. La gasometría arterial mostraba insuficiencia respiratoria $(\mathrm{pH}$ de 7,45, presión arterial de oxígeno de $59 \mathrm{~mm}$ Hg y presión arterial de anhídrido carbónico de $35 \mathrm{~mm} \mathrm{Hg}$ ). En la radiografía de tórax se observaba un patrón micronodular bilateral con conglomerados en ambos lóbulos superiores. La tomografía axial computerizada (TAC) reveló extensa afectación intersticial bilateral en relación con silicosis complicada con formación de grandes masas de fibrosis masiva progresiva (FMP), que presentaban cavitación en su interior no presente en TAC de 9 meses antes, con formaciones mamelonadas en su interior a nivel de lóbulo superior derecho (LSD) (Fig.1). Se realizó una fibrobroncoscopia que no mostró hallazgos significativos y la citología del broncoaspirado no demostró malignidad. En el esputo, el cultivo de lowestein fue negativo y el cultivo para hongos positivo para Aspergillus fumigatus. Una TAC mostró que la lesión cavitada se había reducido de tamaño tras 7 meses de tratamiento con voriconazol. El paciente en estos momentos se encuentra en proceso de valoración para trasplante pulmonar.
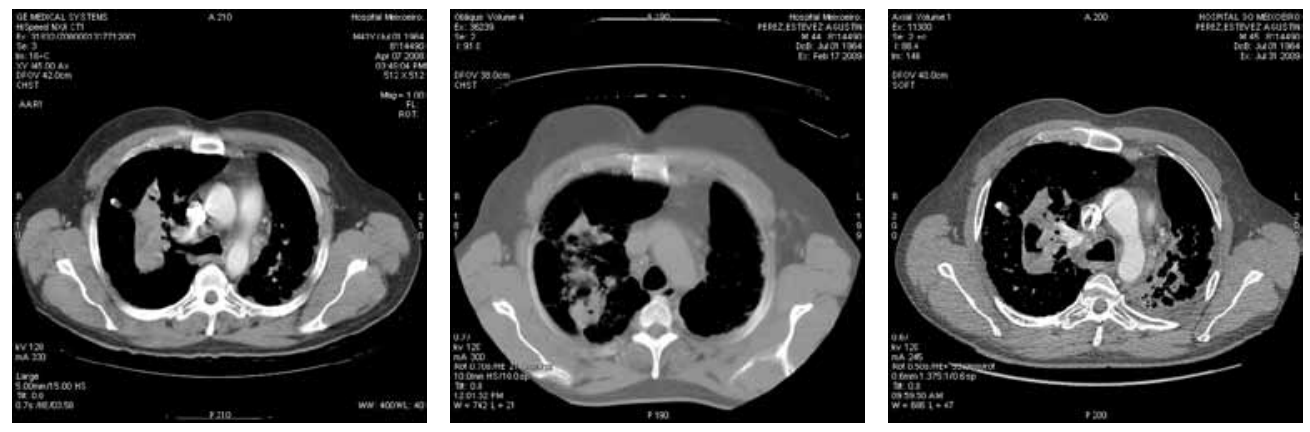

Fig. 1. Áreas de consolidación con progresión a cavitación y formación de aspergiloma a lo largo de 5 meses, sugerentes de APNC (paciente 1).

\section{Caso 2}

Varón de 44 años, trabajador en canteras durante 26 años. Antecedentes de tabaquismo y etilismo. Diagnosticado de tuberculosis pulmonar en 2008 y tratado durante 12 meses. Reingresa un año después por hemoptisis y síndrome general de 2 meses de evolución. En la exploración física destacaba febrícula $\left(37,3^{\circ} \mathrm{C}\right)$, con roncus y crepitantes bilaterales en la auscultación pulmonar. En la analítica sanguínea cabe reseñar leucocitosis (17.000 células/ $/ \mathrm{l}$ con $77 \%$ de neutrófilos, fosfatasa alcalina de $395 \mu / \mathrm{l}$ y VSG de $15 \mathrm{~mm} / \mathrm{h}$. En la radiografía de tórax se apreciaba un patrón intersticial micronodular bilateral difuso y un conglomerado en LSD y lesión cavitaria de pared gruesa en LSI, similar a estudios previos. En la TAC de tórax se apreció extensa afectación intersticial bilateral micronodular con múltiples adenopatías calcificadas, un gran conglomerado en LSD con calcificaciones en su interior similar a estudios previos, apreciándose aumento de la lesión del lóbulo superior izquierdo (LSI) con nueva cavitación, observándose material flotante en su interior compatible con aspergiloma. Las baciloscopias de esputo fueron negativas y el cultivo de esputo positivo para Aspergillus flavus. La serología también fue positiva para Aspergillus flavus y el antígeno galactomanano presentaba positividad débil. Se inició tratamiento con itraconazol. Acude de nuevo a los 3 meses por hemoptisis con persistencia de cultivos positivos para Aspergillus y se cambia el tratamiento a voriconazol con buena evolución clínica hasta el momento actual (3 meses de tratamiento). 


\section{Caso 3}

Varón de 45 años, trabajador en canteras durante 15 años. Diagnosticado de silicosis complicada en FMP. Exfumador desde hace dos años, de 25 paquetes/año. Tuberculosis pulmonar en 2007. Acudió al Servicio de Urgencias en marzo 2009 con hemoptisis amenazante, precedida de tos y expectoración marronácea de 4 semanas de evolución. En la exploración física destacar constantes vitales dentro de la normalidad y roncus bilaterales en la auscultación pulmonar. En la analítica sérica destacar leucocitosis (11.280 células/ $\mu$ l con $80 \%$ de neutrófilos) sin otras alteraciones. La fibrobroncoscopia permitió ver un gran coágulo en bronquio de lóbulo superior derecho. La TAC mostraba afectación intersticial micronodular bilateral y difusa, algunos nódulos calcificados, de predominio en lóbulos superiores y conglomerados que habían aumentado respecto a estudios previos, con aparición de material en su interior, así como engrosamiento pleural nodular y difuso. El cultivo fue positivo para Aspergillus fumigatus en esputo y en aspirado bronquial. En sangre las precipitinas IgG resultaron positivas y el antígeno galactomanano negativo. Se realizó embolización de arterias bronquiales del LSI con control de la hemoptisis y se instauró tratamiento con voriconazol con buena evolución al año de tratamiento con negativización de las precipitinas séricas.

\section{DISCUSIÓN}

Los pacientes con APNC suelen presentar comorbilidad significativa como EPOC, diabetes mellitus, tratamiento crónico con esteroides, VIH, alcoholismo, infección por micobacterias, o edad avanzada ${ }^{5,5}$. La neumoconiosis, que condiciona deterioro de la inmunidad local, es menos frecuente, limitada al 2 y $3 \%$ de los $\operatorname{casos}^{3,7}$. Los 3 casos que presentamos tenían el antecedente de tuberculosis, descrito de manera variable en la literatura (entre el $11 \mathrm{y}$ el $93 \%)^{5,9}$. El tratamiento frecuente con esteroides en el paciente 1 y el antecedente de etilismo en el paciente 2, probablemente colaboraron en la predisposición para APNC. Por otra parte los tres habían sido fumadores importantes, antecedente referido por el $53 \%$ de los pacientes de Nam y col y no mencionado en las otras series ${ }^{9}$. Comparado con la serie de Nam, donde el paciente más joven tenía 48 años y solo el $20 \%$ presen- taron grandes opacidades, los nuestros son más jóvenes y en los 3 casos se objetiva $\mathrm{FMP}^{6}$. Usualmente refieren fiebre, tos y pérdida de peso; y menos frecuentemente disnea o dolor torácico. En nuestro caso se presentaron todos con hemoptisis (Tabla 1), en un caso amenazante, que precisó embolización arterial. Todos presentaban micetoma, cuya presencia oscila entre el 49 y el $90 \%$ de los $\operatorname{casos}^{8,9}$. Los síntomas en APNC usualmente están presentes entre 1 y 6 meses antes de realizarse el diagnóstico, como de hecho ocurrió en nuestros casos, con 1, 2 y 5 meses. Radiológicamente la presencia de Infiltrados en lóbulos superiores, cavitación, opacidades nodulares y engrosamiento pleural son los hallazgos más comunes coincidentes con nuestros $\operatorname{casos}^{10}$.

Es difícil distinguir la APNC de la forma compleja de aspergiloma o APCC: cavidad de pared gruesa con infiltración alrededor, existiendo solapamiento entre ambas entidades $^{8}$. La presencia de algún grado de inmunosupresión y la evolución de escaso meses es más propia de APNC, pero sin una biopsia confimatoria no se puede diferenciar con certeza ambas entidades y quizás deberíamos hablar de aspergilosis pulmonar crónica. La ausencia de enfermedad cavitaria previa como ocurría en nuestros pacientes, permite distinguir ambas entidades de la forma de aspergiloma simple $^{4,6}$. Recientes guías separan ambas entidades en base a diferencias genéticas con defectos en el sistema innato inmune (no estudiados en nuestros casos) en la forma compleja de aspergiloma ${ }^{11}$, así como polimorfismos en lectina de unión a manosa y receptores toll-like se han asociado a esta forma compleja de aspergiloma ${ }^{12}$.

El curso clínico de APNC es menos agresivo que el de la aspergilosis invasiva y por tanto más fácil su diagnóstico diferencial. Los pacientes con silicosis padecen con frecuencia de tuberculosis pulmonar, con síntomas y cambios radiológicos similares a APNC, con lo cual distinguir ambas va ser difícil. La confirmación diagnóstica requiere la demostración histológica de invasión tisular por el hongo y el crecimiento de 
Aspergillus sp en el cultivo, sin embargo la rentabilidad de la biopsia transbronquial y aspirado percutáneo es pobre mientras la biopsia quirúrgica suele desestimarse debido al deterioro de la función respiratoria.

Actualmente el diagnóstico, como se realizó en nuestros pacientes, puede ser hecho en un contexto clínico adecuado en pacientes con radiografía compatible y cultivo positivo para Aspergillus ${ }^{13}$. Las precipitinas, positivas en nuestros 3 casos, aunque no son específicas, son positivas usualmente y su positividad es útil para apoyar el diagnóstico en casos de no aislamiento de Aspergillus. En la actualidad el tratamiento recomendado de APNC, basado en estudios retrospectivos es voriconazol, con el que se puede conseguir una mejoría clínicorradiológica en el $70 \%$ de los pacientes y un control de la enfermedad (control clínicorradiológico y erradicación del hongo) en un 58\% (resultado favorable respecto a controles históricos con itraconazol) ${ }^{14-17}$. Se recomienda la monitorización durante la terapia ${ }^{4}$ cada 3 meses mediante serología (precipitinas) que nosotros realizamos en el caso 3 en el que se produjo negativización a los 12 meses. Son escasos los datos acerca de la duración del tratamiento y la recaída es muy frecuente si se toma de forma no continuada ${ }^{8}$. Nuestros tres pacientes todavía a tratamiento con voriconazol mantienen negatividad en los cultivos para hongos.

La APNC puede ser grave, fatal si no se trata $^{9}$, e incluso puede cursar con síndrome de distress respiratorio agudo ${ }^{18}$. La APNC podría además tener un papel relevante en la mortalidad de los pacientes con silicosis. Así, en el estudio de Lossfova y col $^{19}$ se aprecia cómo las personas que mueren con silicosis, con más probabilidad mueren con micosis pulmonar, mayoritariamente aspergilosis, respecto a las personas sin silicosis (9,5 veces de más probabilidad). Además, en paciente jóvenes como en nuestro primer caso puede contraindicar el trasplante pulmonar.

En conclusión, dado su riesgo potencial y la disponibilidad de tratamientos efectivos si se inician precozmente, debemos considerar el diagnóstico de APNC en pacientes con silicosis, especialmente si aparecen nuevos infiltrados, micetomas, son fumadores y tienen antecedentes de TBP.

\section{BIBLIOGRAFÍA}

1. Hohl TM, Feldmesser M. Aspergillus fumigatus. Principles of pathogenesis and host defense. Eukaryot Cell 2007, 6: 1953-1963.

2. LATGE JP. Aspergillus fumigates and aspergilosis. Clin Microbiol Rev 1999; 12: 310-350.

3. Soubani AO, Chandrasekar PH. The clinical spectrum of pulmonary aspergillosis. Chest 2002; 121: 1988-1999.

4. DenNing DW. Clinical manifestations and diagnostic of chronic pulmonary aspergillosis. UpToDate. Version 18.2. May 2010.

5. Binder RE, Faling LJ, Pugatch RD, Mahasaen MD, SNIDER GL. Chronic necrotizing pulmonary aspergillosis: a discete clinical entity. Medicine 1982: 61: 109-124.

6. Gefter WB, Weingrad TR, Epstein DM, Ochs RH, MILLER WT. Semiinvasive pulmonary aspergillosis: a new look at the spectrum of aspergillosis infectionms of the lung. Radiology 1981; 140: 313-321.

7. Denning DW, Riniotis K, Dobrashian R, Sambatakou H. Chronic cavitary and fibrosing pulmonary and pleural aspergillosis: case series, proposed nomenclature change, and review. Clin Infect Dis 2003; 37 (Suppl. 3) S265-280.

8. Kato T, Usami I, Morita H, Goto M, Hosoda M, NaKamura A, Shima S. Chronic necrotizing pulmonary aspergillosis in pneumoconiosis: clinical and radiologic findings in 10 patients. Chest 2002; 121: 118-127.

9. Nam HS, Jeon K, Um SW, Suh GY, Chung NP, Kim $\mathrm{H}$, Kwon OJ, KOH WJ. Clinical characteristics and treatment outcomes of chronic necrotizing pulmonary aspergillosis: a review of 43 cases. Int J Infect Dis 2009, doi:10.1016/j. ijid.2009.07.011.

10. Franguet T, Muller Nl, Gimenez A, Domingo P, Plaza V, Bordes R. Semiinvasive pulmonary aspergillosis in chronic obstructive pulmonary disease: radiologic and pathologic findings in nine patients. Am J Roentgenol 2000; 174: 51-56.

11. Carbalho A, Pasqualotto AC, Pitzurra L, RomaNI L, Denning DW, Rodrigues F. Polymorfisms in toll-like receptor genes and susceptibility to pulmonary aspergillosis. J Infect Dis 2008; 197: 618-621. 
12. Vald M, Kaur S, Sambatakou H, Madan T, Denning DW, SAMA PU. Distinct alleles of mannose-binding lectin (MBL) and surfactant proteins A (SP-A) in patients with chronic cavitary pulmonary aspergillosis and allergic bronchopulmonary asperillosis. Clin Lab Med 2007; 45: 183-186.

13. Denning DW. Chronic forms of pulmonary aspergillosis. Clin Microbiol Infect 2001; 7 (Suppl. 2): 25-31.

14. Riscili BP, Wood KL. Noninvasive pulmonary aspergillus infections. Clin Chest Med 2009; 30: 315-335.

15. Sherif R, SEgal Bh. Pulmonary aspergillosis: clinical presentation, diagnostic tests, management and complications. Curr Opin Pulm Med 2010; 16: 242-250.
16. Camuset J, Nunes H, Dombret MC, Bergeron A, Henno P, Philippe B et al. Treatment of chronic pulmonary aspergillosis by voriconazole in nonimmunocompromised patients. Chest 2007; 131: 1435-1441.

17. Walsh TJ, Anaissie EJ, Denning DW, Herbrecht R, Kontoyiannis DP, MARR KA et al. Treatment of aspergilosis: clinical practice guidelines of the Infectious Diseases Society of America. Clin Infect Dis 2008; 46: 327-360.

18. YANO S. Acute respiratory distress syndrome due to chronic necrotizing pulmonary aspergillosis. Internal Med 2007; 46: 889-891.

19. Iossifova Y, Bailey R, Wood J, Kreiss K. Concurrent silicosis and pulmonary mycosis at death. Emerg Infect Dis 2010; 16: 318-320. 